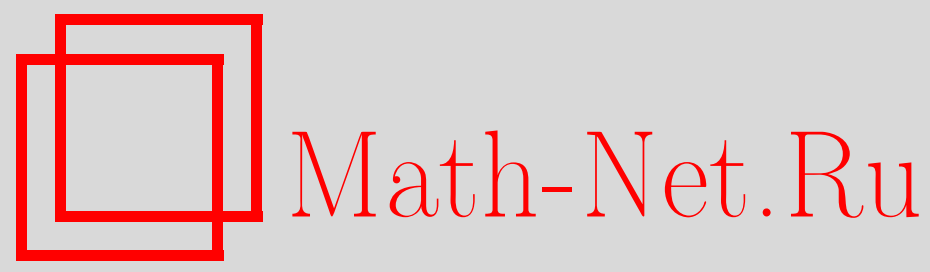

М. Г. Свистула, В. М. Климкин, О поточечном пределе векторных зарядов, обладающих свойством Сакса, Матем. заметки, 2003, том 74, выпуск 3, 407-415

DOI: https://doi.org/10.4213/mzm274

Использование Общероссийского математического портала Math-Net.Ru подразумевает, что вы прочитали и согласны с пользовательским соглашением http://www.mathnet.ru/rus/agreement

Параметры загрузки:

IP : 54.224 .60 .19

26 апреля 2023 г., 15:07:37

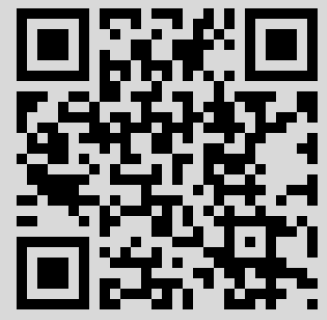




\title{
О ПОТОЧЕЧНОМ ПРЕДЕЛЕ ВЕКТОРНЫХ ЗАРЯДОВ, ОБЛАДАЮЩИХ СВОЙСТВОМ САКСА
}

\author{
В.М. Климкин, М.Г. Свистула
}

\begin{abstract}
Известная теорема о плотности пространства вероятностных зарядов со свойством Сакса в пространстве всех вероятностных зарядов в топологии поточечной сходимости доказана в векторном случае. Новым является рассмотрение равномерного свойства Сакса для семейства зарядов и нахождение достаточных условий для того, чтобы поточечный предел последовательности зарядов обладал свойством Сакса.

Библиография: 11 названий.
\end{abstract}

1. Всюду в дальнейшем: $B$ - булева алгебра с операциями объединения $\vee$, пересечения $\wedge$, разности $\backslash$, дополнения ', отношением порядка $\leqslant$, единицей $e$ и нулем $0 ; Y-$ вещественное линейное нормированноепространство с нормой $\|\cdot\|$ (сокращенно, ЛНП); рассматриваем заряды, определенные на $B$, со значениями в $Y$.

Обозначим через $\mathcal{P}$ множество всех исчерпывающих зарядов, через $\mathcal{L}$ множество исчерпывающих зарядов, обладающих свойством Сакса (определения см. в пункте 2). На $\mathcal{P}$ введем топологию поточечной сходимости. В скалярном случае, а именно для вероятностных зарядов, М. Бхаскара Рао и К. П. С. Бхаскара Рао [1, теорема 6.1] доказали, что $\mathcal{L}$ всюду плотно в $\mathcal{P}$ тогда и только тогда, когда стоуновское пространство $X$ булевой алгебры $B$ является совершенным. В данной статье, модифицируя доказательство теоремы 6.1 [1], мы распространяем этот результат на векторньй случай, когда $Y$ - банахово пространство (см. теорему 1$)$.

Таким образом, в случае совершенного $X$ для любого $\mu \in \mathcal{P}$ существует сеть $\left\{\mu_{\gamma}\right\}_{\gamma \in \Gamma} \subset \mathcal{L}$ такая, что $\mu_{\gamma}(b) \rightarrow \mu(b)$ для любого $b \in B$. В связи с этим возникает вопрос: нельзя ли для заряда $\mu$, не обладающего свойством Сакса, возможно даже имеющего атомы, подобрать последовательность зарядов со свойством Сакса, сходящуюся к $\mu$ поточечно? Используя теорему 2 , получаем отрицательньй ответ на этот вопрос для исчерпьвающего заряда на $f_{1}$-алгебре (следствие 2.7$)$. Помимо этого, теорема 2 представляет самостоятельньй интерес, так как дает достаточные условия для того, чтобы поточечньй предел последовательности зарядов обладал свойством Сакса, а также имеет ряд интересных следствий.

2. Приведем основные определения. Функцию $\mu: B \rightarrow Y$ называем зарядом, если для любых элементов $b_{1}, b_{2} \in B$, где $b_{1} \wedge b_{2}=0$, вьполняется $\mu\left(b_{1} \vee b_{2}\right)=\mu\left(b_{1}\right)+\mu\left(b_{2}\right)$.

Заряд $\mu: B \rightarrow[0,1]$, где $\mu(e)=1$, называем вероятностны.м.

Последовательность $\left\{b_{n}\right\} \subset B$ называем спектром, если $b_{i} \wedge b_{j}=0$ при $i \neq j$. 
Функцию $\mu: B \rightarrow Y$ назьваем мерой, если для любого спектра $\left\{b_{n}\right\} \subset B$, для которого $\bigvee_{n=1}^{\infty} b_{n} \in B$, выполняется $\mu\left(\bigvee_{n=1}^{\infty} b_{n}\right)=\sum_{n=1}^{\infty} \mu\left(b_{n}\right)$.

Говорим, что семейство функций $\left\{\mu_{\gamma}\right\}_{\gamma \in \Gamma}, \mu_{\gamma}: B \rightarrow Y$, равномерно исчерпьваemcя, если для любого спектра $\left\{b_{n}\right\} \subset B$ вьполняется следующее: для любого $\varepsilon>0$ существует $n_{0} \in \mathbb{N}$ такое, что для всех $n>n_{0}$ имеем $\left\|\mu_{\gamma}\left(B_{n}\right)\right\|<\varepsilon$ сразу для всех $\gamma \in \Gamma$. В случае одной функции говорят, что она исчерпывается.

Очевидно, мера на булевой $\sigma$-алгебре, ограниченньй скалярньй заряд на булевой алгебре исчерпьваются.

Пусть $\mu: B \rightarrow Y, b \in B$. Обозначим

$$
\tilde{\mu}(b)=\sup \{\|\mu(a)\|: a \in B, a \leqslant b\} .
$$

Нетрудно показать, что семейство функций $\left\{\mu_{\gamma}\right\}_{\gamma \in \Gamma}$ равномерно исчерпьвается тогда и только тогда, когда семейство функций $\left\{\tilde{\mu}_{\gamma}\right\}_{\gamma \in \Gamma}$ равномерно исчерпьвается [2, гл. $4, \S 1$, теорема 1.1$]$.

Конечньй набор элементов $\left\{b_{1}, \ldots, b_{n}\right\} \subset B$ называем разбиением элемента $b \in B$, если $\bigvee_{i=1}^{n} b_{i}=b$ и $b_{i} \wedge b_{j}=0$ при $i \neq j$.

Говорим, что семейство функций $\left\{\mu_{\gamma}\right\}_{\gamma \in \Gamma}$ обладает равномерным свойством Cакса, если для любого элемента $b \in B$ и для любого $\varepsilon>0$ существует разбиение $\left\{b_{1}, \ldots, b_{n}\right\}$ элемента $b$ такое, что $\tilde{\mu}_{\gamma}\left(b_{i}\right)<\varepsilon$ сразу для всех $\gamma \in \Gamma, i \in 1, \ldots, n$.

Говорим, что семейство функций $\{\mu\}_{\gamma \in \Gamma}$ обладает равномерным слабым свойством Cакса, если для любого элемента $b \in B$ и для любого $\varepsilon>0$ существует разбиение $\left\{b_{1}, \ldots, b_{n}\right\}$ элемента $b$ такое, что $\left\|\mu_{\gamma}\left(b_{i}\right)\right\|<\varepsilon$ сразу для всех $\gamma \in \Gamma, i \in 1, \ldots, n$.

В случае одной функции говорят о свойстве Сакса и слабом свойстве Сакса.

Легко показать, что если семейство зарядов $\{\mu\}_{\gamma \in \Gamma}, \mu_{\gamma}: B \rightarrow Y$, обладает равномерным свойством Сакса, то оно равномерно ограничено. Действительно, существует разбиение $\left\{b_{1}, \ldots, b_{n}\right\}$ элемента $e$ такое, что $\tilde{\mu}_{\gamma}\left(b_{i}\right) \leqslant 1$ сразу для всех $\gamma \in \Gamma, i \in$ $1, \ldots, n$. Тогда для любого $\gamma \in \Gamma$ имеем

$$
\tilde{\mu}_{\gamma}(e) \leqslant \sum_{i=1}^{n} \tilde{\mu}_{\gamma}\left(b_{i}\right) \leqslant n .
$$

Элемент $b \in B, b \neq 0$, назьвается атомом булевой алгебры $B$, если из того, что $a \in B$ и $a \leqslant b$, следует, что $a=0$ или $a=b$.

Алгебра $B$ будет безатомной тогда и только тогда, когда ее стоуновское пространство $X$ является совершенньм, т.е. не имеет изолированньх точек [3, $\S 9]$.

Пусть $\mu: B \rightarrow Y$ - некоторый заряд. Элемент $b \in B$ назьвается атомом $\mu$, если $\mu(b) \neq 0$ и для любого $a \in B$ такого, что $a \leqslant b$, выполняется либо $\mu(a)=0$, либо $\mu(a)=\mu(b)$. Заряд, не имеющий атомов, назьвается неатомическим.

Очевидно, свойство Сакса влечет неатомичность заряда $\mu$. Как показьвает следующий пример, обратное, вообще говоря, не верно.

ПРИМЕР положительного неатомического заряда на $\sigma$-алгебре, не обладающего свойством Сакса. Пусть $\Sigma-\sigma$-алгебра измеримых по Лебегу подмножеств отрезка [0, 1]; пусть, далее, $\mu$ - мера Лебега. Через $\Sigma_{0}$ обозначим класс всех тех множеств из $\Sigma$, для которых $\mu(E)=0$ или $\mu(E)=1$.

Для множества $E \in \Sigma_{0}$ положим

$$
\nu_{0}(E)= \begin{cases}0, & \text { если } \mu(E)=0, \\ 2, & \text { если } \mu(E)=1 .\end{cases}
$$


Очевидно, $\Sigma_{0}$ - подалгебра алгебры $\Sigma$ и $\nu_{0}$ является зарядом на $\Sigma_{0}$. Продолжим $\nu_{0}$ на $\Sigma$ так, чтобы продолжение $\nu$ было зарядом, множество значений которого содержится в замькании множества значений $\nu_{0}[3, \S 47]$. Следовательно, заряд $\nu$ принимает два значения: 0 и 2.

Положим $\varphi=\mu+\nu$. Ясно, что $\varphi$ - неатомический заряд на $\sigma$-алгебре $\Sigma$, не обладающий свойством Сакса: для $\varepsilon=2$ нет разбиения отрезка $[0,1]$, соответствующего определению. Заметим, что $\varphi$ не принимает ни одного значения из отрезка $[1,2]$, в то время как наибольшее значение $\varphi$ на $\Sigma$ равно 3.

Известно, что для меры на булевой $\sigma$-алгебре неатомичность и свойство Сакса эквивалентны.

Неатомичность и свойство Сакса влекут линейную связность образа для широких классов функций [4]. Еще в работах [5], [6] содержался результат о том, что образ неатомической меры $\mu: \Sigma \rightarrow Y$, где $\Sigma-\sigma$-алгебра множеств, является линейно связным множеством. Как показывает приведенный выше пример, образ неатомического заряда на $\sigma$-алгебре не обязан быть линейно связньм. Однако более сильное условие- свойство Сакса - является достаточньм для линейной связности образа заряда на булевой $\sigma$-алгебре (см., например, [4]).

Пусть $\mu: B \rightarrow Y$ - некоторый заряд. Для любого $b \in B$ положим $\|\mu\|(b)=$ $\sup \left\|\sum_{i=1}^{n} \alpha_{i} \mu\left(b_{i}\right)\right\|$, где $\sup$ берется по всевозможным разбиениям $\left\{b_{i}\right\}_{i=1}^{n}$ элемента $b$ и наборам чисел $\left\{a_{i}\right\}_{i=1}^{n}$, где $\left|\alpha_{i}\right| \leqslant 1$. Функция $\|\mu\|: B \rightarrow[0,+\infty]$ назьвается полувариацией $\mu$. Нами будут использованы следующие свойства полувариации: монотонность, равенство $\|\mu\|(b)=\sup \left\{\|f \mu\|(b): f \in Y^{*},\|f\| \leqslant 1\right\}$, оценка $\tilde{\mu}(b) \leqslant\|\mu\|(b) \leqslant 2 \tilde{\mu}(b)$ и тот факт, что если $\mu$ - мера на булевой $\sigma$-алгебре, то $\|\mu\|$ непрерьвна сверху в 0 , т.е. для любой убьвающей последовательности $\left\{b_{n}\right\} \subset B$, где $\bigwedge_{n=1}^{\infty} b_{n}=0$, вьполняется $\lim _{n}\|\mu\|\left(b_{n}\right)=0$. Подробнее о свойствах полувариации см. [2, гл. $\left.2, \S 7\right]$.

Следуя [7], булеву алгебру $B$ будем называть $f_{1}$-алгеброй, если для любых спектров $\left\{a_{n}\right\}$ и $\left\{b_{n}\right\}$ из $B$, для которых $a_{n} \wedge b_{k}=0$ при всех $n, k \in \mathbb{N}$, существуют бесконечное множество индексов $P \subset \mathbb{N}$ и элемент $b \in B$ такие, что $b_{k} \leqslant b$ при всех $k \in P$ и $b \wedge$ $a_{n}=b \wedge b_{k}=0$ при всех $n \in \mathbb{N}$ и $k \in \mathbb{N} \backslash P$.

Очевидно, $\sigma$-алгебра является $f_{1}$-алгеброй. Обратное, как известно, не верно.

3. Далееприведем результаты о разложении, которые известны и доказьвались различными способами (см., например, [8], [9]). Новым здесь является выделение свойства $(r)$, используемого при доказательстве теоремы 1.

ПРЕДЛОЖЕНИЕ 1. Пусть $\mu: B \rightarrow Y-$ мера, где $B$ - булева $\sigma$-алгебра, $Y-$ ЛНП. Тогда справедливо разлохсение

$$
\mu(b)=\mu_{0}+\sum_{i=1}^{\infty} \mu_{i}(b) y_{i}, \quad b \in B
$$

где $\mu_{0}: B \rightarrow Y-$ - натомическая мера, $\mu_{i}: B \rightarrow\{0,1\}-$ двузначные вероятностные мерь, $y_{i} \in Y, i \in \mathbb{N}$. Причем, если $Y$ банахово, то ряд $\sum_{i=1}^{\infty} y_{i}$ обладает свойством $(r)$ : для любого $\varepsilon>0$ существует $n \in \mathbb{N}$ такое, что $\left\|\sum_{i=n+1}^{\infty} \alpha_{i} y_{i}\right\|<\varepsilon$ для всех $\alpha_{i} \in \mathbb{R}$, для которых $\left|\alpha_{i}\right| \leqslant 1, \quad i \in n+1, \ldots, \infty$. 
ДокАЗАТЕЛьСтво. Используя лемму Цорна, легко доказать существование максимального спектра атомов меры $\mu$. Пусть это $\left\{a_{i}\right\}_{i=1}^{\infty} \subset B$. Обозначим $a=e \backslash\left(\bigvee_{i=1}^{\infty} a_{i}\right)$. Положим $\mu_{0}(b)=\mu(b \wedge a), y_{i}=\mu\left(a_{i}\right)$,

$$
\mu_{i}(b)= \begin{cases}1, & \text { если } \mu\left(b \wedge a_{i}\right)=y_{i} \\ 0, & \text { если } \mu\left(b \wedge a_{i}\right)=0\end{cases}
$$

Очевидно, справедливо разложение (1).

Пусть $\left|\alpha_{i}\right| \leqslant 1, i \in \mathbb{N} ; n, m \in \mathbb{N}$. Имеем

$$
\left\|\sum_{i=n+1}^{n+m} \alpha_{i} y_{i}\right\|=\left\|\sum_{i=n+1}^{n+m} \alpha_{i} \mu\left(a_{i}\right)\right\| \leqslant\|\mu\|\left(\bigvee_{i=n+1}^{n+m} a_{i}\right) \leqslant\|\mu\|\left(\bigvee_{i=n+1}^{\infty} a_{i}\right)
$$

Так как полувариация $\|\mu\|$ непрерьвна сверху в 0, то $\|\mu\|\left(\bigvee_{i=n+1}^{\infty} a_{i}\right) \rightarrow 0$ при $n \rightarrow$ $\infty$. Таким образом, вьполняется условие Коши и ряд $\sum_{i=1}^{\infty} \alpha_{i} y_{i}$ сходится, поскольку $Y$ банахово.

Пусть $m \rightarrow \infty$. Тогда

$$
\left\|\sum_{i=n+1}^{\infty} \alpha_{i} y_{i}\right\| \leqslant\|\mu\|\left(\bigvee_{i=n+1}^{\infty} a_{i}\right)
$$

Значит, вьполняется свойство $(r)$.

ПРЕДЛОЖЕНИЕ 2. Пусть $\Sigma-\sigma$-алгебра подмножеств множества $\Omega$, порожденная алгеброй $\mathcal{A}$. Пусть $\mu: \Sigma \rightarrow Y-$ мера, обладающая свойством Сакса. Тогда сужсене $\mu$ на $\mathcal{A}$, обозначаемое $\mu_{\mathcal{A}}: \mathcal{A} \rightarrow Y$, тоже обладает свойством Сакса.

ДокАЗАТЕЛЬСТво. Заметим, что для положительной меры предложение 2 справедливо [1, лемма 3.1$]$.

Известно [10, гл. IV,$\S 10$, лемма 5], что для меры $\mu: \Sigma \rightarrow Y$ существует эквивалентная ей положительная мера $\nu: \Sigma \rightarrow[0,+\infty)$. Эквивалентность здесь может трактоваться как одновременное вьполнение двух условий:

1) для любого $\varepsilon>0$ существует $\delta>0$ такое, что если $E \in \Sigma, \tilde{\mu}(E)<\delta$, то $\nu(E)<\varepsilon$;

2 ) для любого $\varepsilon>0$ существует $\delta>0$ такое, что если $E \in \Sigma, \nu(E)<\delta$, то $\tilde{\mu}(E)<\varepsilon$.

Так как $\mu: \Sigma \rightarrow Y$ обладает свойством Сакса, в силу условия (1) $\nu: \Sigma \rightarrow[0,+\infty)$ тоже обладает свойством Сакса. В силу замечания в начале доказательства $\nu_{\mathcal{A}}: \mathcal{A} \rightarrow$ $[0,+\infty)$ обладает свойством Сакса. Для $\varepsilon>0$ найдем $\delta$ согласно условию (2). Так как $\nu_{\mathcal{A}}$ обладает свойством Сакса, существует разбиение $\left\{b_{1}, \ldots, b_{n}\right\} \subset \mathcal{A}$ элемента $е$ такое, что $\nu\left(b_{i}\right)<\delta, i \in 1, \ldots, n$. Тогда $\tilde{\mu}\left(b_{i}\right)<\varepsilon, i \in 1, \ldots, n$. Очевидно,

$$
\sup \{\|\mu(a)\|: a \in \mathcal{A}, a \leqslant b\} \leqslant \widetilde{\mu}(b), \quad b \in \mathcal{A} .
$$

Значит, $\mu_{\mathcal{A}}$ обладает свойством Сакса. 
ПРЕДЛОЖЕНИЕ 3. Пусть $\mu: B \rightarrow Y-$ исчерпывающий заряд, әде $B$ - булева алгебра, $Y$ - банахово пространство. Тогда справедливо разложсние

$$
\mu(b)=\mu_{0}(b)+\sum_{i=1}^{\infty} \mu_{i}(b) y_{i}, \quad b \in B,
$$

әде $\mu_{0}: B \rightarrow Y-$ исчерпьвающий заряд, обладающий свойством Сакса, $\mu_{i}: B \rightarrow$ $\{0,1\}$ - двузначные вероятностные заряды, $y_{i} \in Y, i \in \mathbb{N} ;$ ряд $\sum_{i=1}^{\infty} y_{i}$ обладает свойством $(r)$.

ДокАЗАТЕЛЬСтво. Пусть $X-$ стоуновское пространство булевой алгебры $B ; \mathcal{A}-$ алгебра открыто-замкнутых множеств; $\Sigma-\sigma$-алгебра, порожденная алгеброй $\mathcal{A} ; T$ : $B \rightarrow \mathcal{A}$ - изоморфизм [3, теорема 8.2].

Очевидно, $\mu T^{-1}: \mathcal{A} \rightarrow Y$-исчерпьвающая мера. Продолжим ее на $\Sigma[11$, теорема 1$]$, а продолжение обозначим $\lambda$. K мере $\lambda: \Sigma \rightarrow Y$ применим разложение (1). Тогда

$$
\lambda(E)=\lambda_{0}(E)+\sum_{i=1}^{\infty} \lambda_{i}(E) y_{i}, \quad E \in \Sigma
$$

где $\lambda_{0}: \Sigma \rightarrow Y$-неатомическая мера, $\lambda_{i}: \Sigma \rightarrow\{0,1\}$ - двузначные вероятностные меры, $y_{i} \in Y, i \in \mathbb{N}$; ряд $\sum_{i=1}^{\infty} y_{i}$ обладает свойством $(r)$.

Так как $\lambda_{0}$ - неатомическая мера на $\sigma$-алгебре $\Sigma$, то $\lambda_{0}$ обладает свойством Сакса на $\Sigma$. В силу предложения $\left.2 \lambda_{0}\right|_{\mathcal{A}}$ тоже обладает свойством Сакса.

Обозначим $\mu_{0}(b)=\lambda_{0}[T(b)], \mu_{i}(b)=\lambda_{i}[T(b)], b \in B, i \in \mathbb{N}$. Очевидно, справедливо разложение (2).

4. Приведенное здесь доказательство теоремы 1 представляет собой модификацию на векторньй случай доказательства теоремы 6.1 работы [1], в которой утверждалось: пусть $B$ - булева алгебра; для того, чтобы множество вероятностных зарядов, обладающих свойством Сакса, было всюду плотным в множестве всех вероятностных зарядов относительно топологии поточечной сходимости, необходимо и достаточно, чтобы стоуновское пространство $X$ булевой алгебры $B$ было совершенным.

Следующее утверждение доказано в работе [1, лемма 6.1].

ПРЕДЛОЖЕНИЕ 4. Пусть $B$ - булева алгебра, стоуновское пространство $X$ которой является совершенным; пусть $b \in B, \quad b \neq 0$. Тогда существует вероятностный заряд $\mu$ на $B$, обладающий свойством Сакса, для которого $\mu(b)=1$.

ТЕОрема 1. Пусть В - булева алгебра, $X$ - ее стоуновское пространство, $Y$ - банахово пространство. Для того чтобы для любого исчерпывающего заряда $\mu: B \rightarrow Y$ существовала сеть исчерпываюших, обладаюших свойством Сакса зарядов $\left\{\mu_{\gamma}\right\}_{\gamma \in \Gamma}, \mu_{\gamma}: B \rightarrow Y$, поточечно сходящаяся $\kappa \mu$, необходимо и достаточно, чтобы $X$ было совериенным.

ДокАЗАТЕЛЬСТво. Достаточность. Пусть $\mu: B \rightarrow Y$ - исчерпьвающий заряд. В силу предложения 3 справедливо разложение (2) с перечисленными в предложении 3 свойствами.

Для любого $i \in \mathbb{N}$ рассмотрим множество $\Gamma_{i}=\left\{b \in B: \mu_{i}(b)=1\right\}$. Положим $\Gamma=$ $\Gamma_{1} \times \Gamma_{2} \times \cdots$. Пусть $\alpha=\left(a_{1}, a_{2}, \ldots\right), \beta=\left(b_{1}, b_{2}, \ldots\right)$, где $\alpha, \beta \in \Gamma$. Полагаем $\alpha \geqslant * \beta$, 
если $a_{i} \leqslant b_{i}$ для любого $i \in \mathbb{N}$. Легко показать, что Г является частично упорядоченным направленным по возрастанию множеством.

Пусть $\beta=\left(b_{1}, b_{2}, \ldots\right), \beta \in \Gamma$. В силу предложения 4 существуют вероятностные, обладающие свойством Сакса, заряды $\mu_{b_{i}}: B \rightarrow[0,1]$ такие, что $\mu_{b_{i}}\left(b_{i}\right)=1$. Положим

$$
\mu_{\beta}(a)=\mu_{0}(a)+\sum_{i=1}^{\infty} \mu_{b_{i}}(a) y_{i}, \quad a \in B
$$

Заметим, что в силу предложения 3 ряд $\sum_{i=1}^{\infty} \mu_{b_{i}}(a) y_{i}$ сходится равномерно относительно $a \in B$. Таким образом, для любого $\varepsilon>0$ существует $n \in \mathbb{N}$ такое, что

$$
\left\|\sum_{i=n+1}^{\infty} \mu_{b_{i}}(a) y_{i}\right\|<\varepsilon
$$

для всех $a \in B$. Учитьвая, что все заряды $\mu_{b_{i}} y_{i}$ исчерпьваются и обладают свойством Сакса, по определению легко доказать исчерпьваемость и свойство Сакса заряда $\sum_{i=1}^{\infty} \mu_{b_{i}} y_{i}$. Тогда $\mu_{\beta}$ - тоже исчерпывающий заряд, обладающий свойством Сакса как сумма зарядов с такими же свойствами.

Покажем, что сеть $\left\{\mu_{\beta}\right\}_{\beta \in \Gamma}$ поточечно сходится к $\mu$. Пусть $b \in B$. Положим $\beta=$ $\left(b_{1}, b_{2}, \ldots\right)$, где $b_{i}=b$, если $b \in \Gamma_{i}$, и $b_{i}=b^{\prime}$, если $b \notin \Gamma_{i}$. Возьмем $\alpha \in \Gamma, \alpha \geqslant * \beta$, где $\alpha=\left(a_{1}, a_{2}, \ldots\right)$. Имеем $\mu_{a_{i}}\left(a_{i}\right)=1$. Так как $a_{i} \leqslant b_{i}$, то в силу монотонности $\mu_{a_{i}}\left(b_{i}\right)=1$. Таким образом, если $\mu_{i}(b)=1$, то $\mu_{a_{i}}(b)=1$; если $\mu_{i}(b)=0$, то $\mu_{a_{i}}(b)=0$. Тогда

$$
\mu_{\alpha}(b)=\mu_{0}(b)+\sum_{i=1}^{\infty} \mu_{a_{i}}(b) y_{i}=\mu_{0}(b)+\sum_{i=1}^{\infty} \mu_{i}(b) y_{i}=\mu(b) .
$$

Таким образом, показано, что для любого $b \in B$ существует $\beta \in \Gamma$ такое, что для всех $\alpha \geqslant \beta$ вьполняется $\mu_{\alpha}(b)=\mu(b)$.

Необходимость. Покажем, что булева алгебра $B$ не имеет атомов. Это, как известно, эквивалентно тому, что $X$ является совершенным. Пусть $b \in B$ и $b \neq 0$. Возьмем $y \in Y$, $y \neq 0$. Легко показать, что существует двузначньй заряд $\mu: B \rightarrow Y$ такой, что $\mu(b)=y$ $[3, \S 47]$. По условию существует сеть обладаюших свойством Сакса зарядов $\left\{\mu_{\gamma}\right\}_{\gamma \in \Gamma}$, $\mu_{\gamma}: B \rightarrow Y$, поточечно сходящаяся к $\mu$. Тогда $\mu_{\gamma}(b) \rightarrow y$. Сушествует $\gamma_{0} \in \Gamma$ такое, что $\left\|\mu_{\gamma_{0}}(b)\right\|>0$. Поскольку заряд $\mu_{\gamma_{0}}$ обладает свойством Сакса, то существует $c \in B$, $c \leqslant b$, для которого $0<\left\|\mu_{\gamma_{0}}(c)\right\|<\left\|\mu_{\gamma_{0}}(b)\right\|$. Значит, $0<c<b$.

СлЕДСТВИЕ 1.1. Пусть $Y$ - произвольное банахово пространство, В - булева алгебра. Следующие три условия әквивалентны:

1) множество всех исчерпывающих, обладающих свойством Сакса зарядов из В в $Y$ всюду плотно в множестве всех исчерпывающих зарядов из $B$ в $Y$ относительно топологии поточечной сходимости;

2) множсество вероятностных, обладаюших свойством Сакса зарядов на В всюду плотно в мнохестве вероятностных зарядов на В относительно топологии поточечной сходимости;

3) стоуновское пространство $Х$ булевой алгебры В является совершенным.

5. Следующая теорема устанавливает достаточные условия того, чтобы семейство зарядов обладало равномерным свойством Сакса. 
Теорема 2. Пусть $M=\left\{\mu_{\gamma}\right\}_{\gamma \in \Gamma}$ - некоторое семейство зарядов, $\mu_{\gamma}: B \rightarrow Y$, В - булева алгебра, $Y$ - ЛНП. Пусть каждый заряд $\mu_{\gamma}$ обладает слабым свойством Сакса. Если семейство М равномерно исчерпывается, то оно обладает равномерным свойством Сакса.

ДокАЗАТЕЛЬСТво. Предположим, что семейство $M$ не обладает равномерным свойством Сакса. Тогда существует $\varepsilon>0$ такое, что для любого разбиения $\left\{b_{1}, \ldots, b_{n}\right\}$ элемента $е$ найдутся $b_{i}$ и $\mu_{\gamma_{i}} \in M$ такие, что $\tilde{\mu}_{\gamma_{i}}\left(b_{i}\right)>\varepsilon$.

Будем говорить, что элемент $c \in B$ обладает свойством (*), если для любого разбиения $\left\{c_{1}, \ldots, c_{m}\right\}$ элемента $c$ найдутся $c_{i}$ и $\mu_{\gamma_{i}} \in M$ такие, что $\tilde{\mu}_{\gamma_{i}}\left(c_{i}\right)>\varepsilon$.

Обозначим $a_{1}=e$. В силу предположения $a_{1}$ обладает свойством $(*)$. Тогда существует заряд $\mu_{\gamma_{1}} \in M$ такой, что $\tilde{\mu}_{\gamma_{1}}\left(a_{1}\right)>\varepsilon$. Отсюда следует существование элемента $p \in B$ такого, что $\left\|\mu_{\gamma_{1}}(p)\right\|>\varepsilon$. Обозначим $q=a_{1} \backslash p$.

Так как заряд $\mu_{\gamma_{1}}$ обладает слабым свойством Сакса, то найдется такое разбиение $\left\{p_{1}, \ldots, p_{n_{1}}\right\}$ элемента $p$, что

$$
\left\|\mu_{\gamma_{1}}\left(p_{i}\right)\right\|<\frac{\varepsilon}{2^{2}}, \quad i \in 1, \ldots, n_{1} .
$$

Итак, $\left\{q, p_{1}, \ldots, p_{n_{1}}\right\}$ - разбиение элемента $a_{1}$. Среди его членов хотя бы один обладает свойством $(*)$. Иначе элемент $a_{1}$ не обладал бы свойством $(*)$.

Если $q$ обладает свойством $(*)$, то положим $a_{2}=q, b_{1}=p$.

Если $q$ не обладает свойством $(*)$, то им обладает некоторьй элемент $p_{i}$. Обозначим $a_{2}=p_{i}$. Положим $b_{1}=p \backslash a_{2}$. Имеем

$$
\left\|\mu_{\gamma_{1}}\left(b_{1}\right)\right\| \geqslant\left\|\mu_{\gamma_{1}}(p)\right\|-\left\|\mu_{\gamma_{1}}\left(a_{2}\right)\right\|>\varepsilon-\frac{\varepsilon}{2^{2}}=\frac{\varepsilon}{2}+\frac{\varepsilon}{2^{2}} .
$$

В любом случае, имеем $\left\|\mu_{\gamma_{1}}\left(b_{1}\right)\right\|>\varepsilon / 2+\varepsilon / 2^{2} ; a_{2}$ обладает свойством $(*)$.

Рассмотрим $a_{2}$ вместо $a_{1}$. Повторим описанную вьше процедуру. Продолжив процесс до бесконечности, получим спектр $\left\{b_{k}\right\} \subset B$ и последовательность зарядов $\left\{\mu_{\gamma_{k}}\right\} \subset$ $M$ такие, что $\left\|\mu_{\gamma_{k}}\left(b_{k}\right)\right\|>\varepsilon / 2+\varepsilon / 2^{k}, k \in \mathbb{N}$. Это противоречит равномерной исчерпываемости семейства $M$.

СлЕДСТВИЕ 2.1. Исчерпывающий заряд $\mu: B \rightarrow Y$, обладающий слабым свойством Сакса, обладает свойством Сакса.

СлЕДСТВИЕ 2.2. Пусть $M=\left\{\mu_{\gamma}\right\}_{\gamma \in \Gamma}, \mu_{\gamma}: B \rightarrow Y,-$ семейство зарядов, каждый из которых обладает свойством Сакса. Если семейство М равномерно исчерпывается, то оно равномерно ограничено.

ДокАЗАТЕЛЬСтво. По теореме 2 семейство $M$ обладает равномерным свойством Сакса и, следовательно, равномерно ограничено.

СлЕДСТВИЕ 2.3. Исчерпывающий заряд $\mu: B \rightarrow Y$ обладает свойством Сакса тогда и только тогда, когда для любого $f \in Y^{*}$ скалярный заряд $f \mu$ обладает свойством Сакса.

ДоказАтЕЛьство. Необходимость очевидна. Докажем достаточность. Рассмотрим семейство $M=\left\{f \mu: f \in Y^{*},\|f\| \leqslant 1\right\}$. Легко проверить, что $M$ равномерно исчерпывается. Тогда по теореме 2 для произвольного $\varepsilon>0$ существует разбиение $\left\{b_{1}, \ldots, b_{n}\right\}$ элемента $e$ такое, что

$$
\lambda_{i}=\sup \left\{\widetilde{f \mu}\left(b_{i}\right): f \in Y^{*},\|f\| \leqslant 1\right\}<\varepsilon, \quad i \in 1, \ldots, n .
$$

Учитьвая свойства полувариации, $\tilde{\mu}\left(b_{i}\right) \leqslant\|\mu\|\left(b_{i}\right) \leqslant 2 \lambda_{i}<2 \varepsilon, i \in 1, \ldots, n$. 
СлЕДСТВИЕ 2.4. Пусть $M=\left\{\mu_{\gamma}\right\}_{\gamma \in \Gamma}, \mu_{\gamma}: B \rightarrow Y$, - некоторая сеть зарядов. Пусть $\mu(b)=\lim \mu_{\gamma}(b)$ для любого $b \in B$. Если каждый заряд $\mu_{\gamma}$ обладает свойством Сакса и семейство М равномерно исчерпывается, то $\mu$ является исчерпывающим зарядом со свойством Сакса.

ДоКАЗАТЕЛЬСТво. Конечная аддитивность и исчерпьваемость $\mu$ сразу следуют из определения, свойство Сакса - из теоремы 2.

Справедлива следующая теорема.

Теорема. Пусть $\left\{\mu_{n}\right\}_{n=1}^{\infty}, \mu_{n}: B \rightarrow Y,-$ некоторая последовательность исчерпываюших зарядов; пусть $B-f_{1}$-алгебра. Пусть $\mu(b)=\lim _{n} \mu_{n}(b)$ для любого $b \in B$. Тогда последовательность $\left\{\mu_{n}\right\}$ равномерно исчерпывается и $\mu$ будет исчерпьвающим зарядом.

ДокАЗАТЕЛьСТво приведено в [7, теорема 2.6] для $f_{1}$-алгебры множеств. Отсюда следует справедливость теоремы и для булевой $f_{1}$-алгебры, так как при изоморфизме между $B$ и алгеброй открыто-замкнутых множеств стоуновского пространства $f_{1}$-свойство и исчерпываемость сохраняются.

СлеДСТВИЕ 2.5. Пусть $\left\{\mu_{n}\right\}_{n=1}^{\infty}, \mu_{n}: B \rightarrow Y,-$ некоторая последовательность зарядов, каждый из которых исчерпывается и обладает свойством Сакса; пусть $B-f_{1}$-алгебра. Пусть $\mu(b)=\lim _{n} \mu_{n}(b)$ для любого $b \in B$. Тогда $\mu$ будет исчерпьвающим зарядом со свойством Сакса.

ДокАЗАТЕЛьство. Последовательность $\left\{\mu_{n}\right\}_{n=1}^{\infty}$ равномерно исчерпьвается. Далее применяем следствие 2.4 .

СлЕДСТВИЕ 2.6. Пусть $\left\{\mu_{n}\right\}_{n=1}^{\infty}, \mu_{n}: B \rightarrow \mathbb{R},-$ некоторая последовательность скалярных зарядов, каждый из которых обладает свойством Сакса; пусть $B$ $f_{1}$-алгебра. Пусть $\mu(b)=\lim _{n} \mu_{n}(b)$ для любого $b \in B$. Тогда $\mu$ будет зарядом со свойством Сакса.

ДокАЗАТЕЛЬСтво. Легко заметить, что $\mu_{n}$ исчерпьвается, $n \in \mathbb{N}$. Остается применить следствие 2.5.

СлЕДСТВИЕ 2.7. Пусть $\mu: B \rightarrow Y$ - исчерпьвающий заряд, не обладающий свойством Сакса; пусть $B-f_{1}$-алгебра. Тогда $\mu$ нельзя представить как поточечный предел некоторой последовательности зарядов, обладающих свойством Сакса.

ДокАЗАТЕЛьСтво. Предположим, что существует последовательность зарядов $\mu_{n}$ : $B \rightarrow Y$, каждый из которых обладает свойством Сакса, и $\mu(b)=\lim _{n} \mu_{n}(b)$ для любого $b \in B$. Возьмем $f \in Y^{*}$. Тогда $f \mu_{n}$ обладает свойством Сакса и $f \mu(b)=\lim _{n} f \mu\left(b_{n}\right)$ для любого $b \in B$. По следствию 2.6 заряд $f \mu$ обладает свойством Сакса. Так как $\mu$ исчерпывается, то по следствию $2.3 \mu$ обладает свойством Сакса, что противоречит условию.

Для скалярного заряда этот результат можно усилить, опустив требование исчерпываемости.

СлЕДСТВИЕ 2.8. Пусть $\mu: B \rightarrow \mathbb{R}-$ скалярный заряд, не обладающий свойством Сакса; пусть $B-f_{1}$-алгебра. Тогда $\mu$ нельзя представить как поточечный предел некоторой последовательности зарядов, обладающих свойством Сакса. 
ДокАЗАТЕЛЬСтво. Предположив противное, получим, что $\mu$ обладает свойством Сакса по следствию 2.6.

\section{Известна следующая}

ТЕОРемА НИКоДима (о сходимости). Пусть $\left\{\mu_{n}\right\}_{n=1}^{\infty}, \mu_{n}: B \rightarrow Y,-$ некоторая последовательность мер; пусть $B-$ булева $\sigma$-алгебра. Пусть $\mu(b)=\lim _{n} \mu_{n}(b)$ для любого $b \in B$. Тогда $\mu$ является мерой.

СлЕДСТВИЕ 2.9. Пусть $\left\{\mu_{n}\right\}_{n=1}^{\infty}, \mu_{n}: B \rightarrow Y,-$ некоторая последовательность неатомических мер; пусть $B-\sigma$-алгебра. Пусть $\mu(b)=\lim _{n} \mu_{n}(b)$ для любого $b \in B$. Тогда $\mu$ является неатомической мерой.

ДокАЗАТЕЛЬСтво. По теореме Никодима о сходимости $\mu$ является мерой. Каждая мера $\mu_{n}$ исчерпывается как мера на $\sigma$-алгебре и обладает свойством Сакса как неатомическая мера на $\sigma$-алгебре.

По следствию 2.5 мера $\mu$ обладает свойством Сакса и, значит, неатомическая.

\section{СПИСОК ЦИТИРОВАННОЙ ЛИТЕРАТУРЫ}

[1] Bhaskara Rao M., Bhaskara Rao K. P. S. Charges on Boolean algebras and almost discrete spaces // Mathematika. 1973. № 20. P. 214-223.

[2] Климкин В. М. Введение в теорию функций множества. Куйбышев: Изд-во Саратовского ун-та, Куйбышевский филиал, 1989.

[3] Сикорский Р. Булевы алгебры. М.: Мир, 1969.

[4] Свистула М. Г. О связности образа неаддитивной функции множества. Деп. в ВИНИТИ № 3765-В98. Самарский госуниверситет, 1998.

[5] Landers D. Connectedness properties of the range of vector and semimeasures // Manuscripta Math. 1973. № 9. P. 105-112.

[6] Constantinescu C. The range of atomless group-valued measures // Comment. Math. Helvetici. 1976. V. 51. № 2. P. 207-213.

[7] Candeloro D. Sui theoremi di Vitali-Hahn-Saks, Dieudonné, e Nikodym // Rend. Circ. Mat. Palermo. 1986. V. 34. № 8. P. 439-445.

[8] Traynor T. Decomposition of group-valued additive set functions // Ann. Inst. Fourier (Grenoble). 1972. V. 22. № 3. P. 131-140.

[9] Weber H. Die atomare Struktur topologischer Boolescher Ringe und s-beschränkter Inhalter // Studia Math. 1982. V. LXXIV. P. 57-81.

[10] Данфорд Н., Шварц Дж. Т. Линейные операторы. Общая теория. М.: ИЛ, 1962.

[11] Кац М. И. О продолжении векторных мер // Сиб. матем. ж. 1972. Т. 13. № 5. С. 1158-1168. 